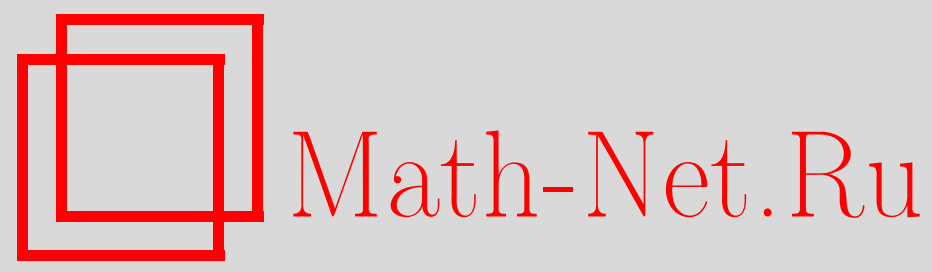

А. Л. Якымив, Тауберова теорема для преобразования Стилтьеса, Матем. заметки, 2003, том 73, выпуск 2, 305-314

DOI: https://doi.org/10.4213/mzm179

Использование Общероссийского математического портала Math-Net.Ru подразумевает, что вы прочитали и согласны с пользовательским соглашением http://www.mathnet.ru/rus/agreement

Параметры загрузки:

IP: 3.91 .87 .62

26 апреля 2023 г., 16:58:38

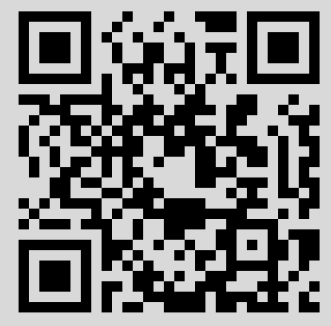


том 73 выПУСК 2 фЕВРАЛЬ 2003

УДК 517.5

\section{ТАУБЕРОВА ТЕОРЕМА ДЛЯ ПРЕОБРАЗОВАНИЯ СТИЛТЬЕСА}

\section{А. Л. Якымив}

В статье при слабых ограничениях на сравниваемые положительные функции из асимптотической эквивалентности на бесконечности их преобразований Стилтьесавыводится асимптотическая эквивалентность на бесконечности самих функций.

Библиографоия: 14 названий.

Функция $f(x)$, определенная и измеримая при $x \in \mathbb{R}_{+}=\{y: y>0\}$, назьвается медленно колеблющейся на бесконечности [1, с. 55], если при $x \rightarrow \infty, y=x+o(x)$ выполнено

$$
f(y)-f(x) \rightarrow 0
$$

Положительная функция $f(x)$, определенная при $x \in \mathbb{R}_{+}$, называется слабо осииллирующей на бесконечности, если функция $\ln f(x)$ медленно колеблется на бесконечности.

Пусть функция $f(x)$ слабо осциллирует на бесконечности. Тогда для нее, как и для всякой $R O$-функции [2, с. 87] при некотором $s>0$ справедливо представление

$$
f(x)=\exp \left(\eta(x)+\int_{s}^{x} \frac{\varepsilon(t)}{t} d t\right), \quad x \geqslant s,
$$

где функции $\eta(x)$ и $\varepsilon(x)$ измеримы и ограничены на $[s, \infty)$.

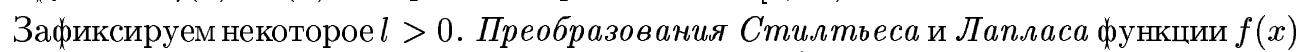
на $\mathbb{R}_{+}$будем обозначать соответственно через $\widetilde{f}(\lambda)$ и $\widehat{f}(\lambda)$ :

$$
\widetilde{f}(\lambda)=\int_{0}^{\infty} \frac{f(x) d x}{(\lambda+x)^{l}}, \quad \widehat{f}(\lambda)=\int_{0}^{\infty} e^{-\lambda x} f(x) d x
$$

(в предположении существования интегралов при $\lambda>0$ ). В настоящей заметке доказывается следующая тауберова теорема.

Работа выполнена при финансовой поддержке Российского фонда фундаментальных исследований, гранты № 00-15-96136, № 03-01-00064 и № 02-01-01080. 
ТЕОремА 1. Пусть функиия $f(x)$ слабо осииллирует на бесконечности; пусть $g(x)=r(x) h(x), x \in \mathbb{R}_{+}$, әде функиия $r(x)$ неотрицательна и монотонна $п р и$ $x \in \mathbb{R}_{+}$, а функиия $h(x)$ слабо осииллирует на бесконечности. Предположим, что для некоторого представления $f(x)$ в форме (1) выполнены неравенства

$$
-1<\inf _{x \geqslant s} \varepsilon(x) \leqslant \sup _{x \geqslant s} \varepsilon(x)<l-1
$$

$u n p u \lambda \rightarrow \infty$

$$
\widetilde{g}(\lambda)=(1+o(1)) \widetilde{f}(\lambda)
$$

Тогда при $x \rightarrow \infty$

$$
g(x)=(1+o(1)) f(x) .
$$

Теорема 1 обобщает соответствующие утверждения из статей [3], [4] (см. также книгу $[5$, гл. Х] $)$. Основное отличие теоремы 1 от предыдущих исследований- отказ от предположения о монотонности функций $f(x)$ и $g(x)$. Тауберова теорема 1 доказывается с помощью некоторых утверждений работы [6]. Из других результатов в этом направлении отметим книгу [7] и работы [8]-[13].

При доказательстве теоремы 1 будут использоваться три леммы, которые приводятся ниже.

Лемма 1. Пусть функиия $f(x)$ слабо осииллирует на бесконечности; пусть $g(x)=r(x) h(x), x \in \mathbb{R}_{+}$, əде функиия $r(x)$ неотрицательна и монотонна $n р и$ $x \in \mathbb{R}_{+}$, а функиия $h(x)$ слабо осииллирует на бесконечности. Предполохим, что для некоторого представления $f(x)$ в форме (1) выполнено неравенство

$$
\inf _{x \geqslant s} \varepsilon(x)>-1
$$

$u n p u \lambda \downarrow 0$

$$
\widehat{g}(\lambda)=(1+o(1)) \widehat{f}(\lambda)
$$

Тогда при $x \rightarrow \infty$ выполнено $g(x)=(1+o(1)) f(x)$.

Лемма 1 следует из теоремы 11 статьи [6].

Согласно [6, с. 177] последовательность функций $\left\{g_{m}(x), m \in \mathbb{N}\right\}$, определенную в $\mathbb{R}_{+}$, назовем асимптотически непрерывной в $\mathbb{R}_{+}$, если для произвольного $x \in \mathbb{R}_{+}$

$$
g_{m}(x)-g_{m}(y) \rightarrow 0, \quad m \rightarrow \infty, \quad y \rightarrow x .
$$

ЛЕмма 2 [6, с. 178]. Пусть последовательность функиий $\left\{g_{m}(x), m \in \mathbb{N}\right\}$ асимптотически непрерывна в $\mathbb{R}_{+}$. Тогда для того чтобы она была предкомпактна в топологии поточечной сходимости, необходимо и достаточно, чтобъ для всякого $x \in \mathbb{R}_{+}$выполнялась оченка

$$
\limsup _{m \rightarrow \infty}\left|g_{m}(x)\right|<\infty .
$$

Функцию $a(x)$, определенную в $\mathbb{R}_{+}$, назовем слабо осииллирующей в нуле, если функция $a(1 / x)$ слабо осциллирует на бесконечности.

Если функция $a(x)$ слабо осциллирует в нуле, то из соотношения (1) следует, что для некоторого $p>0$ при $x \in(0, p]$ справедливо представление

$$
a(x)=\exp \left(\omega(x)+\int_{x}^{p} \frac{\psi(u)}{u} d u\right)
$$

где функции $\omega(x)$ и $\psi(x)$ определены, ограничены и измеримы при $x \in(0, p]$. 
Лемма 3. Пусть

$$
a(x)=x^{k} a_{1}(x)>0, \quad b(x)=x^{k} b_{1}(x)>0, \quad x \in \mathbb{R}_{+},
$$

где $a_{1}(x)$ и $b_{1}(x)$ - убвьвающие функции на $\mathbb{R}_{+}, k>-1$, функция $a(x)$ слабо осииллирует в нуле, причем для некоторого представления $a(x)$ в форме (5) выполнено неравенство

$$
\sup _{0<u \leqslant p} \psi(u)<1 .
$$

Если при $y \rightarrow+\infty$

$$
\widehat{a}(y)=(1+o(1)) \widehat{b}(y),
$$

mo npu $x \downarrow 0$

$$
a(x)=(1+o(1)) b(x)
$$

ДокАЗАТЕльСтво. Предположим противное, а именно: пусть для некоторой последовательности $\left\{x_{m}, m \in \mathbb{N}\right\}$ такой, что $x_{m} \downarrow 0, m \rightarrow \infty$, вьполнено

$$
\frac{b\left(x_{m}\right)}{a\left(x_{m}\right)} \rightarrow c \neq 1, \quad c \in[0, \infty) .
$$

При $\lambda>0$ мы имеем

$$
\widehat{a}\left(\frac{\lambda}{x_{m}}\right)=\int_{0}^{\infty} e^{-\lambda y / x_{m}} a(y) d y=x_{m} a\left(x_{m}\right) \int_{0}^{\infty} \frac{a\left(x_{m} x\right)}{a\left(x_{m}\right)} e^{-\lambda x} d x .
$$

Заметим, что последовательность

$$
f_{m}(x)=\frac{a\left(x_{m} x\right)}{a\left(x_{m}\right)}, \quad x \in \mathbb{R}_{+},
$$

асимптотически непрерывна на $\mathbb{R}_{+}$. В самом деле, при $m \rightarrow \infty, y \rightarrow x>0$

$$
f_{m}(x)-f_{m}(y)=\frac{a\left(x_{m} x\right)-a\left(x_{m} y\right)}{a\left(x_{m}\right)}=\frac{a\left(x_{m} x\right)}{a\left(x_{m}\right)}\left(1-\frac{a\left(x_{m} y\right)}{a\left(x_{m} x\right)}\right)=o(1),
$$

так как

$$
1-\frac{a\left(x_{m} y\right)}{a\left(x_{m} x\right)} \rightarrow 0, \quad m \rightarrow \infty, \quad y \rightarrow x>0,
$$

в силу определения слабо осциллирующей функции, и, как это следует из представления (5),

$$
\frac{a\left(x_{m} x\right)}{a\left(x_{m}\right)}=O(1)
$$

при $m \rightarrow \infty$ и фиксированном $x>0$. Далее, при $t \leqslant p$ для некоторой константы $c_{1}<\infty$ согласно (5) имеем

$$
\frac{a(t x)}{a(t)}=\exp \left(\omega(t x)-\omega(t)+\int_{t x}^{t} \frac{\psi(v)}{v} d v\right) \leqslant \varphi(x)= \begin{cases}c_{1} x^{\alpha}, & 1 \leqslant x \leqslant \frac{p}{t} \\ c_{1} x^{\beta}, & 0<x \leqslant 1\end{cases}
$$


где

$$
\alpha=\sup _{0<v \leqslant p}(-\psi(v)), \quad \beta=-\sup _{0<v \leqslant p} \psi(v)
$$

Положим

$$
g_{m}(x)= \begin{cases}f_{m}(x), & x \leqslant \frac{p}{x_{m}} \\ 0, & x>\frac{p}{x_{m}} .\end{cases}
$$

Так как последовательность $\left\{f_{m}(x), m \in \mathbb{N}\right\}$ асимптотически непрерьвна на $\mathbb{R}_{+}$, то и последовательность $\left\{g_{m}(x), m \in \mathbb{N}\right\}$ тоже асимптотически непрерывна на $\mathbb{R}_{+}$. Согласно лемме 2 без ограничения общности будем считать, что для некоторой непрерьвной функции $f(x)$ на $\mathbb{R}_{+}$

$$
g_{m}(x) \rightarrow f(x), \quad m \rightarrow \infty,
$$

для произвольного $x>0$. Из $(10)$ и определения функций $g_{m}(x)$ следует, что при $m \in \mathbb{N}$ и $x>0$

$$
g_{m}(x) \leqslant \varphi(x)
$$

При этом для всех $\lambda>0$

$$
\widehat{\varphi}(\lambda)<\infty
$$

поскольку по условию леммы 3

$$
\beta=-\sup _{0<v \leqslant p} \psi(v)>-1
$$

Из (11), (12) и (13) по теореме Лебега выводим, что для всех $\lambda>0$

$$
\widehat{g}_{m}(\lambda) \rightarrow \widehat{f}(\lambda)<\infty, \quad m \rightarrow \infty
$$

Далее, при $\lambda>0$

$$
\int_{0}^{\infty} \frac{a\left(x_{m} x\right)}{a\left(x_{m}\right)} e^{-\lambda x} d x=\widehat{g}_{m}(\lambda)+\frac{1}{x_{m} a\left(x_{m}\right)} \int_{p}^{\infty} a(y) e^{-\lambda y / x_{m}} d y .
$$

Согласно $(5)$ при $x \in(0, p]$ для некоторой постоянной $c_{2}>0$ имеем

$$
a(x) \geqslant c_{2} x^{\gamma}
$$

где

$$
\gamma=-\inf _{0<v \leqslant p} \psi(v)
$$

Из (16) следует, что

$$
\begin{aligned}
\frac{1}{x_{m} a\left(x_{m}\right)} \int_{p}^{\infty} a(y) e^{-\lambda y / x_{m}} d y & \leqslant \frac{1}{x_{m} a\left(x_{m}\right)} e^{-\lambda p /\left(2 x_{m}\right)} \int_{p}^{\infty} a(y) e^{-\lambda y /\left(2 x_{m}\right)} d y \\
& =O\left(\frac{e^{-\lambda p /\left(2 x_{m}\right)}}{x_{m} a\left(x_{m}\right)}\right) o(1)=o(1)
\end{aligned}
$$


при $m \rightarrow \infty$. Учитывая (14), из (15) выводим, что

$$
\int_{0}^{\infty} \frac{a\left(x_{m} x\right)}{a\left(x_{m}\right)} e^{-\lambda x} d x \rightarrow \widehat{f}(\lambda) .
$$

Заметим, что при $\lambda>0$

$$
\widehat{b}\left(\frac{\lambda}{x_{m}}\right)=x_{m} a\left(x_{m}\right) \int_{0}^{\infty} \frac{b\left(x_{m} x\right)}{a\left(x_{m}\right)} e^{-\lambda x} d x .
$$

В силу $(6),(9),(17)$ и (18) при $m \rightarrow \infty$

$$
\int_{0}^{\infty} \frac{b\left(x_{m} x\right)}{a\left(x_{m}\right)} e^{-\lambda x} d x \rightarrow \widehat{f}(\lambda), \quad \lambda>0 .
$$

По теореме непрерывности для преобразования Лапласа [14, с. 488, теорема 2а] для произвольного отрезка $A \subseteq \mathbb{R}_{+}$имеем

$$
\int_{A} \frac{b\left(x_{m} x\right)}{a\left(x_{m}\right)} d x \rightarrow \int_{A} f(x) d x .
$$

Зафиксируем произвольное $\delta \in(0,1)$. Покажем, что для некоторой постоянной $c_{3}<\infty$ и всех $m \in \mathbb{N}$

$$
\frac{b_{1}\left(x_{m}\right)}{a_{1}\left(x_{m}\right)} \leqslant c_{3} .
$$

В самом деле, согласно (20) существует константа $c_{4}<\infty$ такая, что для всех $m \in \mathbb{N}$ выполнена оценка

$$
\int_{1-\delta}^{1} \frac{b\left(x_{m} x\right)}{a\left(x_{m}\right)} d x=\int_{1-\delta}^{1} \frac{b_{1}\left(x_{m} x\right) x^{k}}{a_{1}\left(x_{m}\right)} d x \leqslant c_{4}
$$

откуда

$$
\frac{b_{1}\left(x_{m}\right)}{a_{1}\left(x_{m}\right)} \int_{1-\delta}^{1} x^{k} d x \leqslant c_{4}
$$

или же вьполнено (21) с константой

$$
c_{3}=c_{4}\left(\int_{1-\delta}^{1} x^{k} d x\right)^{-1}
$$

Заметим, что

$$
\begin{aligned}
\frac{b\left(x_{m}\right)}{a\left(x_{m}\right)} & =\frac{1}{\delta} \int_{1-\delta}^{1} \frac{b\left(x_{m}\right)}{a\left(x_{m}\right)} d x \\
& =\frac{1}{\delta}\left(\int_{1-\delta}^{1} \frac{b\left(x_{m}\right)-b\left(x_{m} x\right)}{a\left(x_{m}\right)} d x+\int_{1-\delta}^{1} \frac{b\left(x_{m} x\right)}{a\left(x_{m}\right)} d x\right) \\
& =\frac{1}{\delta}\left(\int_{1-\delta}^{1} \frac{b\left(x_{m} x\right)}{a\left(x_{m}\right)} d x+\int_{1-\delta}^{1} \frac{b_{1}\left(x_{m}\right)-b_{1}\left(x_{m} x\right) x^{k}}{a_{1}\left(x_{m}\right)} d x\right) \\
& \leqslant \frac{1}{\delta}\left(\int_{1-\delta}^{1} \frac{b\left(x_{m} x\right)}{a\left(x_{m}\right)} d x+\frac{b_{1}\left(x_{m}\right)}{a_{1}\left(x_{m}\right)} \int_{1-\delta}^{1}\left(1-x^{k}\right) d x\right) \\
& =\frac{1}{\delta} \int_{1-\delta}^{1} \frac{b\left(x_{m} x\right)}{a\left(x_{m}\right)} d x+\frac{(k+1) \delta-\left(1-(1-\delta)^{k+1}\right)}{(k+1) \delta} \cdot \frac{b_{1}\left(x_{m}\right)}{a_{1}\left(x_{m}\right)}
\end{aligned}
$$


Из (20), (21) и (22) следует, что

$$
\limsup _{m \rightarrow \infty} \frac{b\left(x_{m}\right)}{a\left(x_{m}\right)} \leqslant \frac{1}{\delta} \int_{1-\delta}^{1} f(x) d x+c_{3} \frac{(k+1) \delta-\left(1-(1-\delta)^{k+1}\right)}{(k+1) \delta}
$$

Устремляя в правой части выписанного неравенства $\delta$ к нулю и пользуясь непрерывностью $f(x)$, получаем, что

$$
\limsup _{m \rightarrow \infty} \frac{b\left(x_{m}\right)}{a\left(x_{m}\right)} \leqslant f(1)
$$

Точно так же, используя интегрирование от 1 до $1+\delta$, получаем, что

$$
\liminf _{m \rightarrow \infty} \frac{b\left(x_{m}\right)}{a\left(x_{m}\right)} \geqslant f(1)
$$

Стало быть, существует

$$
\lim _{m \rightarrow \infty} \frac{b\left(x_{m}\right)}{a\left(x_{m}\right)}=f(1) .
$$

Вспоминая (11) и определение функций $g_{m}(x)$, убеждаемся в том, что

$$
f(1)=1 .
$$

Итак, мы получили, что

$$
\frac{b\left(x_{m}\right)}{a\left(x_{m}\right)} \rightarrow 1, \quad m \rightarrow \infty .
$$

Последнее соотношение противоречит (8). Лемма 3 доказана.

ДокАЗАТЕЛЬСТво тЕоРЕмЫ 1. Покажем, что для некоторого $p<1 / s$ при $\lambda \in(0, p]$ функция $\widehat{f}(\lambda)$ представима в виде

$$
\widehat{f}(\lambda)=\frac{1}{\lambda} \exp \left(\int_{1 / p}^{1 / \lambda} \frac{\varepsilon(t)}{t} d t+\xi(\lambda)\right)
$$

где $\varepsilon(t)$ - функция из представления $f(x)$ в форме $(1)$, а $\xi(\lambda)$ ограничена при $\lambda \in(0,1 / p]$. Доопределим $\varepsilon(x)$ и $\eta(x)$ нулем при $x<s$. Так как

$$
\inf _{x \geqslant s} \varepsilon(x)>-1
$$

то $\widehat{f}(\lambda) \rightarrow \infty, \lambda \downarrow 0$. Поэтому при $\lambda \downarrow 0$ вьполнено

$$
\begin{aligned}
\widehat{f}(\lambda) & =\int_{0}^{\infty} e^{-\lambda x} \exp \left(\eta(x)+\int_{0}^{x} \frac{\varepsilon(t)}{t} d t\right) d x+O(1) \\
& =(1+o(1)) \int_{0}^{\infty} e^{-\lambda x} \exp \left(\eta(x)+\int_{0}^{x} \frac{\varepsilon(t)}{t} d t\right) d x
\end{aligned}
$$


Поэтому достаточно получить такое же представление для

$$
\int_{0}^{\infty} e^{-\lambda x} \exp \left(\eta(x)+\int_{0}^{x} \frac{\varepsilon(t)}{t} d t\right) d x
$$

Заметим, что

$$
\begin{aligned}
\int_{0}^{\infty} e^{-\lambda x} \exp \left(\eta(x)+\int_{0}^{x} \frac{\varepsilon(t)}{t} d t\right) d x & =\frac{1}{\lambda} \int_{0}^{\infty} e^{-u} \exp \left(\eta\left(\frac{u}{\lambda}\right)+\int_{0}^{u / \lambda} \frac{\varepsilon(t)}{t} d t\right) d u \\
& =\frac{1}{\lambda} \exp \left(\int_{0}^{1 / \lambda} \frac{\varepsilon(t)}{t} d t\right) I(\lambda)
\end{aligned}
$$

где

$$
I(\lambda)=\int_{0}^{\infty} e^{-u} \exp \left(\eta\left(\frac{u}{\lambda}\right)+\int_{1 / \lambda}^{u / \lambda} \frac{\varepsilon(t)}{t} d t\right) d u
$$

Для доказательства (23) достаточно показать, что для некоторых постоянных $c_{1}, c_{2}$ при $\lambda \in(0,1 / s]$ выполнены оценки

$$
0<c_{1} \leqslant I(\lambda) \leqslant c_{2}<\infty
$$

Представим интеграл $I(\lambda)$ в виде суммы интегралов $I_{1}(\lambda)$ и $I_{2}(\lambda)$ по $u$ соответственно от 0 до 1 и от 1 до $\infty$ :

$$
I(\lambda)=I_{1}(\lambda)+I_{2}(\lambda)
$$

При $\lambda \in(0,1 / s]$ мы имеем

$$
I_{1}(\lambda)=\int_{0}^{1} e^{-u} \exp \left(\eta\left(\frac{u}{\lambda}\right)-\int_{u / \lambda}^{1 / \lambda} \frac{\varepsilon(t)}{t} d t\right) d u \leqslant e^{c_{3}} \int_{0}^{1} \exp (-\alpha(-\ln u)) d u
$$

где

$$
c_{3}=\sup _{v \geqslant 0} \eta(v), \quad \alpha=\inf _{t \geqslant s} \varepsilon(t)
$$

Поэтому при $\lambda \in(0,1 / s]$ вьполнено

$$
I_{1}(\lambda) \leqslant e^{c_{3}} \int_{0}^{1} u^{\alpha} d u=\frac{e^{c_{3}}}{\alpha+1}
$$

так как согласно неравенствам (2) $\alpha>-1$. Далее,

$$
\begin{aligned}
I_{2}(\lambda) & =\int_{1}^{\infty} e^{-u} \exp \left(\eta\left(\frac{u}{\lambda}\right)+\int_{1 / \lambda}^{u / \lambda} \frac{\varepsilon(t)}{t} d t\right) d u \\
& \leqslant e^{c_{3}} \int_{1}^{\infty} e^{-u} \exp (\beta \ln u) d u=e^{c_{3}} \int_{1}^{\infty} e^{-u} u^{\beta} d u<\infty
\end{aligned}
$$

где

$$
\beta=\sup _{t \geqslant s} \varepsilon(t)
$$


Кроме того,

$$
I_{1}(\lambda) \geqslant e^{c_{4}} \int_{0}^{1} e^{-u} \exp (-\beta(-\ln u)) d u=e^{c_{4}} \int_{0}^{1} e^{-u} u^{\beta} d u>0
$$

и

$$
I_{2}(\lambda) \geqslant e^{c_{4}} \int_{1}^{\infty} e^{-u} \exp (\alpha \ln u) d u=e^{c_{4}} \int_{1}^{\infty} e^{-u} u^{\alpha} d u>0
$$

где

$$
c_{4}=\inf _{v \geqslant 0} \eta(v)
$$

Из соотношений (25)-(29) следует (24). Далее, покажем, что $\widehat{f}(\lambda)$ слабо осциллирует в нуле. Пусть $\mu, \lambda \downarrow 0, \mu / \lambda \rightarrow 1$. Тогда

$$
\begin{aligned}
\widehat{f}(\lambda) & =\int_{0}^{\infty} e^{-\lambda x} f(x) d x=\frac{\mu}{\lambda} \int_{0}^{\infty} e^{-\mu u} f\left(\frac{\mu}{\lambda} u\right) d u \\
& =(1+o(1)) \int_{0}^{\infty} e^{-\mu u} f\left(\frac{\mu}{\lambda} u\right) d u \\
& =(1+o(1))\left(\widehat{f}(\mu)+\int_{0}^{\infty} e^{-\mu u} f(u)\left(1-\frac{f(\mu u / \lambda)}{f(u)}\right) d u\right) .
\end{aligned}
$$

Поэтому достаточно показать, что

$$
\int_{0}^{\infty} e^{-\mu u} f(u)\left(1-\frac{f(\mu u / \lambda)}{f(u)}\right) d u=o(\widehat{f}(\mu))
$$

при $\lambda, \mu \downarrow 0, \lambda / \mu \rightarrow 1$. Покажем это. Заметим, прежде всего, что для произвольного фиксированного $t>0$ вьполнено

$$
\begin{aligned}
\int_{0}^{t} e^{-\mu u} f(u)\left(1-\frac{f(\mu u / \lambda)}{f(u)}\right) d u & =\int_{0}^{t} e^{-\mu u} f(u) d u-\int_{0}^{t} e^{-\mu u} f\left(\frac{\mu}{\lambda} u\right) d u \\
& =O(1)=o(\widehat{f}(\mu))
\end{aligned}
$$

при $\lambda, \mu \downarrow 0, \lambda / \mu \rightarrow 1$. Зафиксируем произвольное $\varepsilon>0$. Для этого $\varepsilon$ подберем такие $t>0$ и $\delta>0$, чтобы при $u \geqslant t$ и $|\mu / \lambda-1| \leqslant \delta$ было выполнено неравенство

$$
\left|1-\frac{f(\mu u / \lambda)}{f(u)}\right| \leqslant \varepsilon
$$

Тогда в силу (31) и (32) имеет место оценка

$$
\limsup _{\mu \downarrow 0, \lambda / \mu \rightarrow 1} \frac{1}{\widehat{f}(\mu)}\left|\int_{0}^{\infty} e^{-\mu u} f(u)\left(1-\frac{f(\mu u / \lambda)}{f(u)}\right) d u\right| \leqslant \varepsilon
$$


Так как $\varepsilon$-произвольное положительное число, из последнего неравенства следует (30). Как известно [8, с. 41], преобразование Стилтьеса есть двойное преобразование Лапласа, а именно, при $\lambda>0$

$$
\widetilde{f}(\lambda)=\int_{0}^{\infty} a(x) e^{-x \lambda} d x, \quad \widetilde{g}(\lambda)=\int_{0}^{\infty} b(x) e^{-x \lambda} d x
$$

где

$$
a(x)=\frac{x^{l-1} \widehat{f}(x)}{\Gamma(l)}, \quad b(x)=\frac{x^{l-1} \widehat{g}(x)}{\Gamma(l)} .
$$

Как было показано ранее, $\widehat{f}(x)$ слабо осциллирует в нуле, стало быть, слабо осциллирует в нуле и функция $a(x)$, причем из (23) следует, что при $x \in(0,1 / p]$ вьполняются равенства

$$
\begin{aligned}
a(x) & =x^{l-2} \exp \left(\int_{1 / p}^{1 / x} \frac{\varepsilon(t)}{t} d t+\xi(x)-\ln \Gamma(l)\right) \\
& =x^{l-2} \exp \left(\int_{x}^{p} \frac{\varepsilon(1 / u)}{u} d u+\xi(x)-\ln \Gamma(l)\right)=\exp \left(\int_{x}^{p} \frac{\psi(u)}{u} d u+\omega(x)\right),
\end{aligned}
$$

где

$$
\psi(x)=2-l+\varepsilon\left(\frac{1}{x}\right), \quad \omega(x)=\xi(x)-\ln \Gamma(l)+(2-l) \ln p .
$$

Заметим, что согласно (2)

$$
\sup _{0<x \leqslant p} \psi(x)=2-l+\sup _{0<x \leqslant p} \varepsilon\left(\frac{1}{x}\right)<1
$$

Применяя лемму 3 , получаем, что при $x \downarrow 0$

$$
a(x)=(1+o(1)) b(x)
$$

откуда в силу (34) следует, что при $x \downarrow 0$

$$
\widehat{f}(x)=(1+o(1)) \widehat{g}(x)
$$

Из последнего соотношения и леммы 1 следует, что при $x \rightarrow \infty$

$$
f(x)=(1+o(1)) g(x) .
$$

Теорема 1 доказана. 


\section{СПИСОК ЦИТИРОВАННОЙ ЛИТЕРАТУРЫ}

[1] Постников А. Г. Тауберова теория и ее применения. М.: Наука, 1979.

[2] Сенета Е. Правильно меняющиеся функции. М.: Наука, 1985.

[3] Келдыш М. В. Об одной тауберовой теореме // Тр. МИАН. 1951. Т. 38. С. 77-86.

[4] Мацаев В. И., Палант Ю. А. О распределении спектра полиномиального операторного пучка // Докл. АН АрмССР. 1966. Т. 42. № 5. С. 836-845.

[5] Костюченко А.Г., Саргсян И.С. Распределение собственных значений. М.: Наука, 1979.

[6] Якымив А. Л. Асимптотика вероятности продолжения критических ветвящихся процессов Беллмана-Харриса // Тр. МИАН. 1986. Т. 177. С. 177-205.

[7] Pilipoviĉ S., Stancoviĉ B., Takaĉi A. Asymptotic behaviour and Stieltjes transformation of distributions. Teubner-Texte zur Mathematik. V. 116. Leipzig: Teubner, 1990.

[8] Белогрудь В.П. Об одной тауберовой теореме // Матем. заметки. 1974. Т. 15. № 2. C. $187-190$.

[9] Султанаев Я. Т. О спектре неполуограниченных обыкновенных дифференциальных операторов. Дисс. ... к.ф̆.-м.н. М.: МГУ, 1974.

[10] Nicoliĉ-Despotoviĉ D., Pilipoviĉ S. Tauberian theorems for the distributional Stieltjes Transformation // Intern. J. Math. Math. Sci. 1986. V. 9. № 3. P. 531-524.

[11] Pilipoviĉ S. On the quasiasymptotic behaviour of the Stieltjes transformation of distributions // Publ. Inst. Math. 1987. V. 40. P. 143-152.

[12] Selander T. Bilateral Tauberian theorems of Keldysh type // Ark. Mat. 1963. V. 5. № 6 . P. 85-96.

[13] Станкович Б. Абелевы и тауберовы теоремы для преобразования Стилтьеса обобщенных функций // УМН. 1985. V. 40. № 4. Р. 91-103.

[14] Феллер В. Введение в теорию вероятностей и ее приложения. Т. 2. М.: Мир, 1984.

Математический институт им. В. А. Стеклова РАН

Поступило

E-mail: arsen@mi.ras.ru

28.11.2001 